\title{
Correspondence
}

\section{Pleural effusion associated with urinary tract obstruction}

Sir,-We read with interest the recently published case report by Leung and Oill (Thorax 1981;36:632-3) of a pleural effusion associated with urinary tract obstruction. The authors concluded that because the BUN of the pleural fluid was $19 \mathrm{mg} \%$ and a transudate, as compared to the perinephric urine which had a BUN of $139 \mathrm{mg} \%$, the pleural fluid was probably not urine but a transudate secondary to compression of transdiaphragmatic lymphatics. They went on to postulate that most or all cases of pleural effusion, in association with urinary tract obstruction as reported in the literature, are probably not urine but transudates, probably secondary to similar mechanisms. We have recently reviewed the radiographic manifestations of urinothorax (Baron et al. AJR 1981; 137:37-41) as well as the biochemical features (Stark et al, in manuscript). Biochemical analysis of the pleural fluid was available in three patients. The pleural BUN was measured in only one patient, and was $55 \mathrm{mg} \%$ as compared to a serum BUN of $29 \mathrm{mg} \%$. In all three of our cases there was a marked elevation in the pleural creatinine as compared to the serum creatinine. The pleural creatinine was $10 \cdot 3,8 \cdot 6$, and $14 \cdot 6$ compared to the serum creatinines (drawn within $24 \mathrm{hrs}$ of obtaining the pleural fluid) of $1 \cdot 2,3 \cdot 3$, and $12.5 \mathrm{mg} \%$ respectively. To confirm that elevation of creatinine is not common for pleural effusions generally, we obtained paired pleural and serum creatine determinations on 66 patients with pleural effusions of varying aetiology. In this group we found that irrespective of the aetiology, the pleural creatinine was nearly identical to the serum creatinine in all cases. The greatest excess in pleural fluid creatinine concentration was $0.6 \mathrm{mg}$ per dl greater than the serum value. These biochemical data support radiological evidence that the pleural fluid in "urinothorax" is indeed urine.
Another case, not cited by the authors, (Lahiry et al. $\frac{\overline{\bar{S}}}{\overline{\mathrm{S}}}$ $J$ Trauma 1978;18:608-10), describes a pleural effusion $\overparen{Ð}$ associated with a urinoma in which the pleural fluid BUN was $120 \mathrm{mg} \%$ despite a normal serum BUN. Moreover, $\infty$ there was the appearance of Indigo carmine in the pleural $\overrightarrow{0}$ fluid and urine after an intravenous injection.

We wish to point out, therefore, that pleural effusions $\vec{\omega}$ associated with urinary tract obstruction can represent $\Omega$ urine, and that the case reported by Leung and Oill, in $\overrightarrow{\vec{x}}$ which the pleural fluid was not urine, probably represents the exception rather than the rule.

$$
\begin{array}{r}
\text { JEFFREY G SHANES } \\
\text { ROBERT M SENIOR } \\
\text { Department of Medicine } \\
\text { The Jewish Hospital of St Louis } \\
\text { Washington University } \\
\text { DAVID D STARK } \\
\text { Department of Radiology } \\
\text { University of California } \\
\text { RICHARD L BARON } \\
\text { Re } \\
\text { Department of Radiology } \\
\text { Hospital of the University of Pennsylvania }
\end{array}
$$

\section{Notice}

Second International Workshop on Respiratory Psychophysiology

Hamburg, West Germany, 25-26 June 1982

Discussion during the meeting will deal with hypotheses $\overrightarrow{\overrightarrow{0}}$ and present knowledge concerning psychophysiological 3 and psychosomatic aspects of human breathing, respiration and respiratory disorders. For further details, pleaseco. write to Dr Rainer Richter, Department of Psychosomatic Medicine, University Clinic Eppendorf, Pav 23, Martinistr 52, D-2000 Hamburg 20. 